\title{
Specialized System Identification for Parafoil and Payload Systems
}

\author{
Michael Ward* and Mark Costelloı \\ Georgia Institute of Technology, Atlanta, Georgia 30332 \\ and \\ Nathan Slegers \\ University of Alabama in Huntsville, Huntsville, Alabama 35899
}

DOI: $\underline{10.2514 / 1.53364}$

\begin{abstract}
There are a number of peculiar aspects to parafoil and payload systems that make it difficult to apply conventional system identification procedures used for aerospace systems. Parafoil and payload systems are unique because typically there is very little sensor information available, the sensors that are available are separated from the canopy by a complex network of flexible rigging, the systems are very sensitive to wind and turbulence, the systems exhibit a number of nonlinear behaviors, and the systems exhibit a high degree of variability from flight to flight. The current work describes a robust system identification procedure developed to address the specific difficulties posed by airdrop systems. By employing a two-phase approach that separately considers atmospheric winds estimation and aerodynamic coefficient estimation, a nonlinear, 6-degree-of-freedom dynamic simulation model is generated using only Global Positioning System data from the flight test. The key to this approach is the use of a simplified aerodynamic representation of the canopy, which requires identification of only the steady-state response to control input to completely define the dynamic model. The proposed procedure is demonstrated by creating a simulation model using Global Positioning System data from actual flight tests. To validate the procedure, the dynamic response of the simulation model is then compared to inertial measurement unit data that were not used in any way to develop the simulation model, with excellent results.
\end{abstract}

\section{Nomenclature}

$A, B, C=$ Lamb's coefficients for apparent mass, $\mathrm{kg}$

$b \quad=$ canopy span, $\mathrm{m}$

$d \quad=$ canopy arc radius, $\mathrm{m}$

$[I] \quad=$ identity matrix

$\left[I_{T}\right] \quad=$ total system inertia matrix $\mathrm{kg} \cdot \mathrm{m}^{2}$

$L, M, N=$ components of moment vector in the body reference frame, $\mathrm{N} \cdot \mathrm{m}$

$m \quad=\quad$ total system mass, $\mathrm{kg}$

$P, Q, R=$ Lamb's coefficients for apparent inertia, $\mathrm{kg} \cdot \mathrm{m}^{2}$

$p, q, r=$ angular velocity components in the body reference frame, $\mathrm{rad} / \mathrm{s}$

$\tilde{p}, \tilde{q}, \tilde{r}=$ angular velocity components in the canopy reference frame, $\mathrm{rad} / \mathrm{s}$

$S=$ aerodynamic reference area, $\mathrm{m}^{2}$

$\left[S_{\omega}^{B}\right] \quad=$ skew symmetric cross product operator for angular velocity expressed in frame $B$, rad/s

$\left[S_{x, y}^{B}\right] \quad=$ skew symmetric cross product operator for distance vector from system $x$ to $y$ in frame $b, \mathrm{~m}$

$T_{B, i}=$ transformation matrix from body frame to $i$ th canopy element frame

$T_{I B} \quad=$ transformation matrix from inertial frame to body frame

$u, v, w=$ velocity components of mass center in the body reference frame, $\mathrm{m} / \mathrm{s}$

$\tilde{u}, \tilde{v}, \tilde{w}=$ velocity components with respect to the air, $\mathrm{m} / \mathrm{s}$

$X, Y, Z=$ components of force in the body reference frame, $\mathrm{N}$

Received 8 December 2010; revision received 16 June 2011; accepted for publication 6 September 2011. Copyright () 2011 by Michael Ward. Published by the American Institute of Aeronautics and Astronautics, Inc., with permission. Copies of this paper may be made for personal or internal use, on condition that the copier pay the $\$ 10.00$ per-copy fee to the Copyright Clearance Center, Inc., 222 Rosewood Drive, Danvers, MA 01923; include the code 0731-5090/12 and \$10.00 in correspondence with the CCC.

${ }^{*}$ Graduate Research Assistant, School of Aerospace Engineering. Member AIAA.

†Sikorsky Associate Professor, School of Aerospace Engineering. Associate Fellow AIAA.

${ }^{\ddagger}$ Assistant Professor, Department of Mechanical and Aerospace Engineering. Member AIAA.

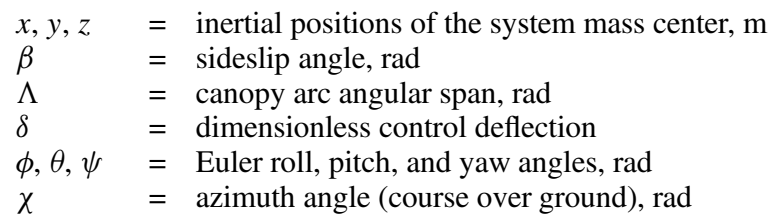

Subscripts

$\begin{array}{ll}A & =\text { aerodynamic } \\ B & =\text { body frame } \\ C & =\text { canopy } \\ i & =i \text { th element of the canopy } \\ P & =\text { payload } \\ W & =\text { weight }\end{array}$

\section{Introduction}

$\mathbf{M}$ ODERN engineering of airdrop systems leans heavily on flight dynamic modeling and simulation to predict a multitude of drop events virtually so that guidance, navigation, and control (GNC) software can be developed and tested in a cost efficient manner. For the GNC development effort to be successful, reasonably accurate dynamic models that exhibit similar nonlinear behaviors to the actual airdrop systems are required. These models are obtained by performing system identification on flight-test data of the system under development.

The traditional aerodynamic model used for dynamic simulation of aircraft is created using a linearized representation of the system dynamics near trimmed flight [1]. The main difficulty with this representation is the need to identify a large number of aerodynamic coefficients. The coefficients are often correlated with one another and a large number of them can typically be neglected, though the particular coefficients that can be neglected may vary from aircraft to aircraft. The problem is difficult enough for fixed wing aircraft and rotorcraft, but it becomes even more difficult for airdrop systems. The primary differences that cause these difficulties are the limited number of control channels available to excite the system dynamics (the only means of control typically used on a parafoil is differential or symmetric deflection of the trailing edge), the sensitivity of these systems to wind (due to low flight speed and low mass to volume 
ratios), and the limited amount and quality of available sensor data (due to the typical constraint that sensors must be confined to the payload which is separated from the canopy by a complex network of flexible rigging).

A variety of methods have been developed for air vehicle system identification $[2,3]$. The two methods that are best suited to the current problem are the output error method (OEM), which is the most common method for parameter identification from noisy measurements, and identification through an extended Kalman filter, which is commonly used when there is both measurement and process noise. These two methods can also be combined to form the filter error method.

A number of efforts have applied aerodynamic parameter identification to airdrop systems [4-11]. Jann et al. describe the development of a highly instrumented parafoil and payload research platform, ALEX, incorporating a Global Positioning System (GPS) receiver, magnetometers, rate gyros, accelerometers, air data probes, and video cameras to address the problem of generating enough data for system identification [4]. With this system, they performed an extensive investigation into the problem of aerodynamic parameter identification for parafoil and payload systems [5-7]. Their initial approach was to develop 3- and 4-degree-of-freedom (DOF) models (the extra DOF was added to account for significant bank angles). These models required a small number of coefficients to be identified and good results were obtained [5]. Efforts to identify the extra coefficients needed for a 6-DOF model were more difficult, and a creative approach to generating the coefficients by applying lifting line theory to an arc anhedral wing was used to generate initial estimates of all of the parameters as well as approximate relationships for correlated coefficients [6]. Finally, an 8-DOF model was developed incorporating the relative motion of the payload using the same approach [7]. In each case, the use of the air data probe allowed the estimation of wind and turbulence so that the OEM could be applied on data with the effect of the wind removed. Even so, the process required frequent intervention to produce good results [7]. Hur and Valasek describe the development of another highly instrumented platform including an inertial measurement unit (IMU), flow sensors, and even accelerometers installed in the midsection of the parafoil [8]. They demonstrated the identification of a linear 8-DOF model from simulation data using an observer/ Kalman filter identification method described by Valasek and Chen [9]. Kothandaraman and Rotea described the use of a computationally efficient method to identify coefficients for a 6-DOF circular parachute model assuming perfect knowledge of the winds [10]. Yakimenko and Statnikov presented a method for identifying aerodynamic coefficients of an 8-DOF parafoil model using a multicriteria optimization method beginning with a parameter space investigation to help address the problems of local minima and infeasible regions in the parameter space [11]. In particular, they noted that most of the aerodynamic coefficients in a general model of a parafoil and payload system seem to be strongly correlated, there are many sets of parameters that produce equally good reproductions of the observed flight data, and the additional degrees of freedom accounting for payload motion helped to match the natural eigenvalues of the roll, pitch, and yaw measurements but did not affect the matching of the system trajectory.

All of these works approach the problem in slightly different ways, but a common thread among them is the necessity of using a highly instrumented platform specialized for the system identification task in order to obtain sufficient data for successful aerodynamic parameter identification. In contrast to these specialized platforms, fielded airdrop systems have only a single GPS receiver [12-14]. This is desirable to reduce cost and complexity, especially because the GPS receiver does not require any preflight calibration. The current work describes a reliable system identification procedure that requires only GPS data, which would allow the system identification task to be performed using the same hardware intended for the field. This is highly advantageous in streamlining the development cycle of an airdrop system. Of course, if it is possible to obtain measurements in addition to GPS, it would not make sense to restrict the system identification to GPS data alone. In this case, the system identification procedure described here could be used to develop an approximate model based on GPS alone which could then be refined with whatever additional sensor information is available. Depending on the available sensor data, this refinement could be performed using similar methods to the existing airdrop identification methods mentioned previously [4-11]. Another possibility is that the model made from GPS data using the proposed procedure could be simply augmented to capture unmodeled dynamics observed in the additional sensor channels using more general system identification techniques such as those recently developed by Majji et al. $[15,16]$.

A number of techniques for deriving particular aspects of the flight dynamic characteristics of a parafoil and payload system using only GPS data have been described [12-14]. The restricted amount of sensor information available leads to a very restricted dynamic model of the system, normally a linear 3-DOF representation. Typically, only the steady-state longitudinal characteristics are modeled by developing a simple model of air speed and glide path angle as a function of symmetric brake input; lateral dynamics consist of modeling the system heading rate dynamics as a first- or secondorder filter of the asymmetric brake input (trailing-edge deflection is commonly referred to as a brake input). In contrast, the current work seeks to develop a 6-DOF, nonlinear simulation model using only GPS data. This type of simulation model is beneficial during GNC development to ensure that the guidance and control algorithms are robust to nonlinear behaviors that may not be captured by simpler representations.

The key to developing a 6-DOF model from GPS data alone is the use of an aerodynamic model which requires the identification of a minimum number of coefficients. An alternative to applying the standard aerodynamic model of a point with force and moment coefficients was used by Slegers and Costello to explore control issues with parafoil and payload systems [17]. Their model makes use of five discrete canopy elements producing only lift and drag, so aerodynamic moments and side force are produced by the orientation of the elements and their displacement from the center of mass. This is highly attractive for system identification with minimal sensor data because only lift and drag coefficients need to be estimated to obtain a complete nonlinear 6-DOF simulation model, and these coefficients can be estimated entirely from steady-state information.

The first step in the proposed system identification procedure is designing input sequences to ensure that high-quality data can be obtained in a timely manner. Second, the vehicle airspeed is estimated by extracting the wind vector from the GPS data. Once the effect of the wind vector is removed, steady-state longitudinal and lateral control response data in the form of lift, drag, and turn rate vs symmetric and asymmetric brake deflection is extracted. This steadystate data represent the highest quality information obtained from the flight tests because it has been averaged over a large number of measurements to remove the effects of sensor error and turbulence. All of the parameters of the simulation model affecting the steadystate control response are then determined through nonlinear regression to match the steady-state control response data as opposed to attempting to match flight data directly. At this stage, a complete 6DOF nonlinear model has been created based only on GPS data.

The proposed system identification procedure is applied to a small parafoil and payload system. The system has an IMU in addition to a GPS receiver. This allows the time histories of payload orientation and angular rates from flight data to be compared with simulation results for the model derived using only GPS data. These comparisons are used to ensure that an adequate modeling of the transient characteristics of a parafoil and payload system can be achieved using a model derived from GPS data alone. Finally, the identified simulation model is validated against data from an additional flight test that was not used for system identification.

\section{Parafoil and Payload Dynamic Model}

Figure 1 shows a schematic of a parafoil and payload system. With the exception of movable parafoil brakes, the parafoil canopy is considered to be a fixed shape. The combined system of the parafoil canopy and the payload are modeled with $6 \mathrm{DOF}$, including three 


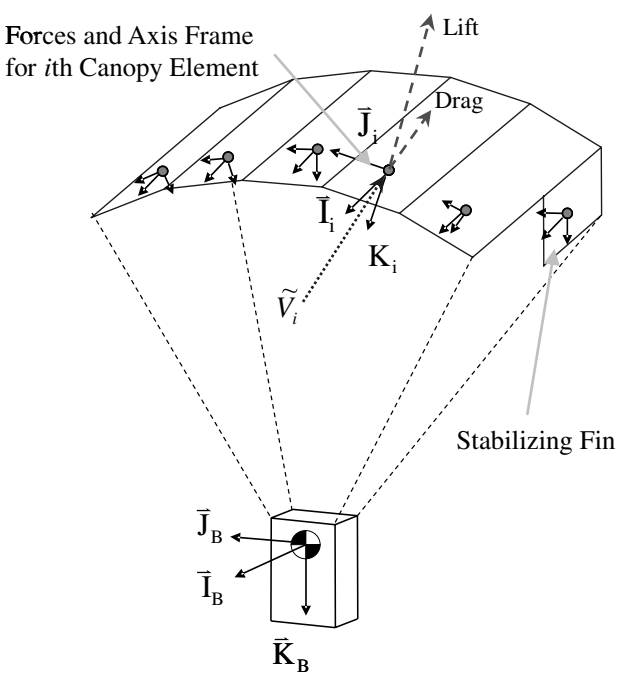

Fig. 1 Parafoil and payload schematic.

inertial position components of the total system mass center as well as the three Euler orientation angles. The canopy is modeled as seven flat panels, five main panels representing the ram-air canopy and two fins under the outboard edges of the canopy. The fins provide additional directional stability to provide a more realistic transient turn rate response as well as roll and yaw damping to delay the onset of turning into a deep spiral. Each panel produces only lift and drag, so side force and aerodynamic moments are developed from the orientation and displacement of the panels relative to the center of mass. Suspension lines are not modeled explicitly.

Derivations of the equations of motion for a 6-DOF rigid aircraft are readily available in the flight dynamic literature [1]. The current discussion focuses only on the aspects of the equations of motion which are unique to parafoil and payload systems and to the particular aerodynamic modeling approach used in this work. The kinematic equations for the parafoil and payload system are provided in Eqs. (1) and (2). The shorthand notation for trigonometric functions is employed where $\sin (x) \equiv s_{x}, \cos (x) \equiv c_{x}$, and $\tan (x) \equiv t_{x}$ :

$$
\begin{gathered}
\left\{\begin{array}{c}
\dot{x} \\
\dot{y} \\
\dot{z}
\end{array}\right\}=\left[T_{I B}\right]^{T}\left\{\begin{array}{c}
u \\
v \\
w
\end{array}\right\} \\
\left\{\begin{array}{c}
\dot{\phi} \\
\dot{\theta} \\
\dot{\psi}
\end{array}\right\}=\left[\begin{array}{ccc}
1 & s_{\phi} t_{\theta} & c_{\phi} t_{\theta} \\
0 & c_{\phi} & -s_{\phi} \\
0 & s_{\phi} / c_{\theta} & c_{\phi} / c_{\theta}
\end{array}\right]\left\{\begin{array}{c}
p \\
q \\
r
\end{array}\right\}
\end{gathered}
$$

The matrix $\left[T_{I B}\right]$ represents the transformation matrix from the inertial reference frame to the body reference frame:

$$
T_{I B}=\left[\begin{array}{ccc}
c_{\theta} c_{\psi} & c_{\theta} s_{\psi} & -s_{\theta} \\
s_{\phi} s_{\theta} c_{\psi}-c_{\phi} s_{\psi} & s_{\phi} s_{\theta} s_{\psi}+c_{\phi} c_{\psi} & s_{\phi} c_{\theta} \\
c_{\phi} s_{\theta} c_{\psi}+s_{\phi} s_{\psi} & c_{\phi} s_{\theta} s_{\psi}-s_{\phi} c_{\psi} & c_{\phi} c_{\theta}
\end{array}\right]
$$

The dynamic equations are formed by summing forces and moments about the system center of gravity (CG), both in the body reference frame, and equating to the time derivative of linear and angular momentum, respectively:

$$
\begin{gathered}
m\left(\left\{\begin{array}{c}
\dot{u} \\
\dot{v} \\
\dot{w}
\end{array}\right\}+\left[S_{\omega}^{B}\right]\left\{\begin{array}{c}
u \\
v \\
w
\end{array}\right\}\right)=\left\{\begin{array}{c}
X_{W} \\
Y_{W} \\
Z_{W}
\end{array}\right\}+\left\{\begin{array}{c}
X_{A M} \\
Y_{A M} \\
Z_{A M}
\end{array}\right\} \\
+\left\{\begin{array}{c}
X_{A, P} \\
Y_{A, P} \\
Z_{A, P}
\end{array}\right\}+\sum_{i}\left\{\begin{array}{c}
X_{A, i} \\
Y_{A, i} \\
Z_{A, i}
\end{array}\right\}
\end{gathered}
$$

$$
\begin{gathered}
{\left[I_{T}\right]\left\{\begin{array}{c}
\dot{p} \\
\dot{q} \\
\dot{r}
\end{array}\right\}+S_{\omega}^{B}\left[I_{T}\right]\left\{\begin{array}{c}
p \\
q \\
r
\end{array}\right\}=\left\{\begin{array}{c}
L_{A M} \\
M_{A M} \\
N_{A M}
\end{array}\right\}+\left[S_{\mathrm{cg}, M}^{B}\right]\left\{\begin{array}{c}
X_{A M} \\
Y_{A M} \\
Z_{A M}
\end{array}\right\}} \\
+\left[S_{\mathrm{cg}, P}^{B}\left\{\begin{array}{c}
X_{A, P} \\
Y_{A . P} \\
Z_{A . P}
\end{array}\right\}+\sum_{i}\left[S_{\mathrm{cg}, i}^{B}\right]\left\{\begin{array}{c}
X_{A, i} \\
Y_{A . i} \\
Z_{A . i}
\end{array}\right\}\right.
\end{gathered}
$$

$S$ is the skew symmetric operator, used to express the cross product of two vectors in terms of the components of the vectors in a specified frame. For example, if the vectors $\mathbf{a}, \mathbf{b}$, and $\mathbf{c}$ are expressed in terms of their components in frame B,

$$
\begin{aligned}
\mathbf{c} & =\mathbf{a} \times \mathbf{b} \Leftrightarrow\left\{\begin{array}{c}
c_{x} \\
c_{y} \\
c_{z}
\end{array}\right\}=\left[\begin{array}{ccc}
0 & -a_{z} & a_{y} \\
a_{z} & 0 & -a_{x} \\
-a_{y} & a_{x} & 0
\end{array}\right]\left\{\begin{array}{l}
b_{x} \\
b_{y} \\
b_{z}
\end{array}\right\} \\
& =\left[S_{a}^{B}\right]\left\{\begin{array}{c}
b_{x} \\
b_{y} \\
b_{z}
\end{array}\right\}
\end{aligned}
$$

Forces appearing in Eq. (4) have contributions from weight, aerodynamic loads on the canopy and payload, and apparent mass (AM). The weight contribution is given in Eq. (7):

$$
\left\{\begin{array}{l}
X_{W} \\
Y_{W} \\
Z_{W}
\end{array}\right\}=m g\left\{\begin{array}{c}
-s_{\theta} \\
s_{\phi} c_{\theta} \\
c_{\phi} c_{\theta}
\end{array}\right\}
$$

Defining the transformation from the body frame to the frame attached to the $i$ th element of the canopy as $\left[T_{B, i}\right]$ and defining the wind speed and wind heading in the inertial frame as $V_{W}$ and $\psi_{W}$, respectively, the "aerodynamic" velocity of the $i$ th element is given by Eq. (8). The term aerodynamic velocity is used to refer to the velocity of a particular point on the system with respect to the air. The aerodynamic velocity of the payload is given by the same equation with the body frame to element transformation equal to the identity matrix:

$$
\left\{\begin{array}{c}
\tilde{u}_{i} \\
\tilde{v}_{i} \\
\tilde{w}_{i}
\end{array}\right\}=\left[T_{B, i}\right]\left(\left\{\begin{array}{c}
u \\
v \\
w
\end{array}\right\}-\left[S_{\mathrm{cg}, i}^{B}\right]\left\{\begin{array}{c}
p \\
q \\
r
\end{array}\right\}-\left[T_{I B}\right]\left\{\begin{array}{c}
V_{W, x} \\
V_{W, y} \\
V_{W, z}
\end{array}\right\}\right)
$$

The aerodynamic forces on the canopy elements are expressed in terms of lift and drag coefficients, which are functions of the angle of attack of each element, $\alpha_{i}=\tan ^{-1}\left(\tilde{w}_{i} / \tilde{u}_{i}\right)$, as shown in Eqs. (9) and (10). Equation (11) defines the canopy aerodynamic forces in the body reference frame. For the outboard panels, the lift and drag coefficients are modified by the control deflections, $\delta_{i}$. In addition to the linear term, a term proportional to the third power of control deflection was added to account for the rapid changes in lift and drag coefficient observed for large brake deflections:

$$
\begin{gathered}
C_{L, i}=C_{L 0, i}+C_{L A, i} \alpha_{i}+C_{L B, i} \delta_{i}+C_{L B 3, i} \delta_{i}^{3} \\
C_{D, i}=C_{D 0, i}+C_{D A 2, i} \alpha_{i}^{2}+C_{D B, i} \delta_{i}+C_{D B 3, i} \delta_{i}^{3}
\end{gathered}
$$

$$
\begin{aligned}
& \left\{\begin{array}{c}
X_{A, i} \\
Y_{A . i} \\
Z_{A . i}
\end{array}\right\}=-\frac{1}{2} \rho S_{i}\left[T_{B, i}\right]^{T}\left(C_{L, i} \sqrt{\tilde{u}_{i}^{2}+\tilde{w}_{i}^{2}}\left\{\begin{array}{c}
\tilde{w}_{i} \\
0 \\
-\tilde{u}_{i}
\end{array}\right\}\right. \\
& \left.+C_{D, i} \sqrt{\tilde{u}_{i}^{2}+\tilde{v}_{i}^{2}+\tilde{w}_{i}^{2}}\left\{\begin{array}{c}
\tilde{u}_{i} \\
\tilde{v}_{i} \\
\tilde{w}_{i}
\end{array}\right\}\right)
\end{aligned}
$$


The aerodynamic force on the payload consists entirely of profile drag and is given by Eq. (12):

$$
\left\{\begin{array}{c}
X_{A, P} \\
Y_{A . P} \\
Z_{A . P}
\end{array}\right\}=-\frac{1}{2} \rho S_{P} C_{D, P} \sqrt{\tilde{u}_{P}^{2}+\tilde{v}_{P}^{2}+\tilde{w}_{P}^{2}}\left\{\begin{array}{c}
\tilde{u}_{P} \\
\tilde{v}_{P} \\
\tilde{w}_{P}
\end{array}\right\}
$$

Parafoils with small mass-to-volume ratios can experience large forces and moments from accelerating fluid. These are termed AM effects. A precise accounting of these effects can substantially complicate the dynamic equations, but it is possible to obtain a good approximation of the effects with only a few terms. The approximate forms used for the AM forces and moments are given in Eqs. (13-15). Parametric approximations given by Lissaman and Brown are used to determine the AM and inertia coefficients in Eq. (15) [18]:

$$
\begin{gathered}
\left\{\begin{array}{c}
X_{A M} \\
Y_{A M} \\
Z_{A M}
\end{array}\right\}=-\left[I_{A M}\right]\left(\left\{\begin{array}{c}
\dot{\tilde{u}} \\
\dot{\tilde{v}} \\
\dot{\tilde{w}}
\end{array}\right\}-\left[S_{\mathrm{cg}, M}^{B}\right]\left\{\begin{array}{c}
\dot{\tilde{p}} \\
\dot{\tilde{q}} \\
\dot{\tilde{r}}
\end{array}\right\}\right) \\
\left\{\begin{array}{c}
L_{A M} \\
M_{A M} \\
N_{A M}
\end{array}\right\}=-\left[I_{A I}\right]\left\{\begin{array}{c}
\dot{\tilde{p}} \\
\dot{\tilde{q}} \\
\tilde{r}
\end{array}\right\} \\
{\left[I_{A M}\right]=\left[\begin{array}{ccc}
A & 0 & 0 \\
0 & B & 0 \\
0 & 0 & C
\end{array}\right], \quad\left[I_{A I}\right]=\left[\begin{array}{ccc}
P & 0 & 0 \\
0 & Q & 0 \\
0 & 0 & R
\end{array}\right]}
\end{gathered}
$$

The dynamic equations of motion are found by substituting all forces and moments into Eqs. (4) and (5), resulting in the matrix solution in Eqs. (16-18):

$$
\begin{aligned}
& {\left[\begin{array}{cc}
m[I]+\left[I_{A M}\right] & -\left[I_{A M}\right]\left[S_{\mathrm{cg}, M}^{B}\right] \\
{\left[S_{\mathrm{cg}, M}^{B}\right]\left[I_{A M}\right]} & {\left[I_{T}\right]+\left[I_{A I}\right]-\left[S_{\mathrm{cg}, M}^{B}\right]\left[I_{A M}\right]\left[S_{\mathrm{cg}, M}^{B}\right]}
\end{array}\right]\left\{\begin{array}{c}
\dot{u} \\
\dot{v} \\
\dot{w} \\
\cdots \\
\dot{p} \\
\dot{q} \\
\dot{r}
\end{array}\right\}} \\
& =\left[\begin{array}{c}
B_{1} \\
\cdots \\
B_{2}
\end{array}\right] \\
& B_{1}=-m\left[S_{\omega}^{B}\right]\left\{\begin{array}{c}
u \\
v \\
w
\end{array}\right\}+\left\{\begin{array}{c}
X_{W} \\
Y_{W} \\
Z_{W}
\end{array}\right\}+\left\{\begin{array}{c}
X_{A, P} \\
Y_{A, P} \\
Z_{A, P}
\end{array}\right\}+\sum_{i}\left\{\begin{array}{c}
X_{A, i} \\
Y_{A, i} \\
Z_{A, i}
\end{array}\right\} \\
& B_{2}=-\left[S_{\omega}^{B}\right]\left[I_{T}\right]\left\{\begin{array}{c}
p \\
q \\
r
\end{array}\right\}+\left[S_{\mathrm{cg}, P}^{B}\right]\left\{\begin{array}{c}
X_{A, P} \\
Y_{A, P} \\
Z_{A, P}
\end{array}\right\}+\sum_{i}\left[S_{\mathrm{cg}, i}^{B}\right]\left\{\begin{array}{c}
X_{A, i} \\
Y_{A, i} \\
Z_{A, i}
\end{array}\right\}
\end{aligned}
$$

Equation (16) represents a set of coupled, nonlinear differential equations. The matrix on the left-hand side of Eq. (16) is a function of the mass and geometry properties of the parafoil. The geometry of the parafoil is assumed to be fixed, so this matrix is constant and only needs to be inverted once at the beginning of the simulation. With specified initial conditions, the states can be numerically integrated forward in time.

\section{System Identification Method}

The proposed system identification method is summarized in Fig. 2. The main idea is to match the steady-state response to control input extracted from segments of flight-test data. To this end, the majority of the procedure is dedicated to processing flight-test data to obtain high-quality estimates of the steady-state lift, drag, and turn rate response to control input. Aerodynamic coefficients in the simulation model are then used to match these extracted steady-state characteristics rather than the flight-test data directly. For this procedure, validation refers to checking the identified model against data from a flight that was not used during the matching process.

\section{A. Input Sequences}

The structure of the input sequence is critical to obtaining highquality data for system identification. The sequence must ensure that both steady-state and transient characteristics can be estimated, and the sequences for each flight must be arranged so that the loss of any single flight is not detrimental to the system identification effort.

The input sequences were designed primarily based on the need to generate high-quality estimates of the steady-state characteristics of the parafoil and payload system. This requires relatively long periods of constant control input at various combinations of symmetric and asymmetric brake deflection. Given the total amount of flight testing time available, the number of control input combinations to be tested was chosen so that at some point during flight testing, each control input would be held for two to four times the amount of time necessary for the system to reach steady state. This ensures that there will be enough data available after the system has reached equilibrium to average the steady-state response over a series of measurements to reduce the influence of sensor errors and turbulence. In addition, the order of the control input combinations are selected so that the system will receive doublet maneuvers in both symmetric and asymmetric brakes as it transitions from one control combination to the next. This ensures the system is excited sufficiently so that the transient characteristics can be determined. The actual control sequences used in flight test are shown in the results section.

\section{B. Extracting Steady-State Lift, Drag, and Turn Rate}

The first step in the system identification process is wind estimation. The ground track velocity measured with GPS can be decomposed into an airspeed vector $\left(\boldsymbol{V}_{0}\right)$ and wind vector $\left(\boldsymbol{V}_{w}\right)$ as shown in Fig. 3 . Since the ground track velocity vector is the only measured quantity, solution of the vector diagram is an underdetermined problem. Solution of the vector diagram requires a series of measurements at different azimuth angles.

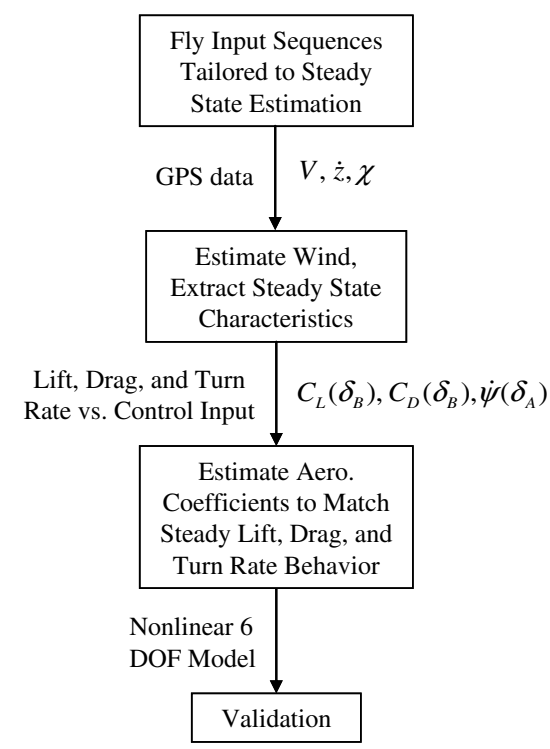

Fig. 2 System identification procedure. 


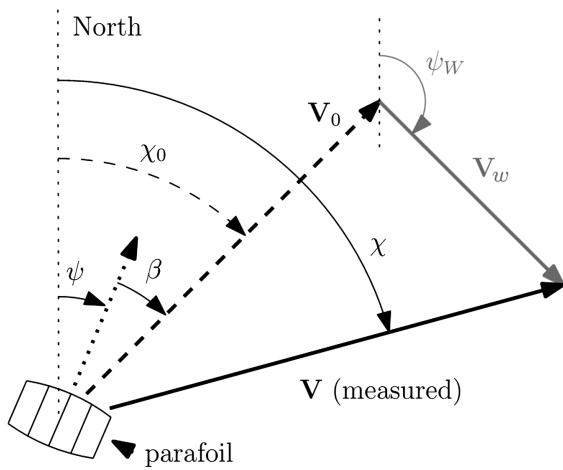

Fig. 3 Decomposing measured velocity vector.

The approach taken is to break the flight into segments of constant control deflection, then assume a constant forward airspeed $\left(V_{0}\right)$ and wind vector for each segment. This leaves three unknowns (two wind vector components and the airspeed) for each of the flight segments. For a series of $n$ measurements taking during a constant control segment, the $x$ (north) and $y$ (east) components of the $i$ th measurement of ground track velocity can be decomposed into a wind component and an airspeed component:

$$
\begin{gathered}
\dot{x}_{i}=V_{W, X}+V_{0} \cos \left(\psi_{i}\right), \quad \dot{y}_{i}=V_{W, Y}+V_{0} \sin \left(\psi_{i}\right) \\
V_{0}^{2}=\left(\dot{x}_{i}-V_{W, X}\right)^{2}+\left(\dot{y}_{i}-V_{W, Y}\right)^{2} \\
=V_{i}^{2}+V_{W}^{2}-2\left(\dot{x}_{i} V_{W, x}+\dot{y}_{i} V_{W, Y}\right)
\end{gathered}
$$

For the constant control segment, the airspeed and wind vector are assumed constant, so these terms can be removed from Eq. (20) by subtracting the expected values:

$$
\begin{aligned}
& V_{0}^{2}-E\left(V_{0}^{2}\right)=0=V_{i}^{2}-E\left(V_{i}^{2}\right)-2\left(\left(\dot{x}_{i}-E\left(\dot{x}_{i}\right)\right) V_{W, x}\right. \\
& \left.\quad+\left(\dot{y}_{i}-E\left(\dot{y}_{i}\right)\right) V_{W, Y}\right)
\end{aligned}
$$

where the expected values of the measured quantities are estimated by sample means:

$$
E\left(V_{i}^{2}\right)=\mu_{V 2}, \quad E\left(\dot{x}_{i}\right)=\mu_{\dot{x}}, \quad E\left(\dot{y}_{i}\right)=\mu_{\dot{y}}
$$

The problem of estimating the wind vector components for the constant control segment can now be cast as a linear regression problem as shown in Eq. (23). Once the wind vector components have been calculated, the airspeed can be estimated for each measurement according to Eq. (24). The steady-state airspeed of the vehicle for a given control deflection is obtained as the average of the airspeed estimates over a particular constant control flight segment:

$$
\begin{gathered}
{\left[\begin{array}{cc}
\dot{x}_{1}-\mu_{\dot{X}} & \dot{y}_{1}-\mu_{\dot{Y}} \\
\vdots & \vdots \\
\dot{x}_{n}-\mu_{\dot{X}} & \dot{y}_{n}-\mu_{\dot{Y}}
\end{array}\right]\left\{\begin{array}{l}
V_{W, X} \\
V_{W, Y}
\end{array}\right\}=\frac{1}{2}\left\{\begin{array}{c}
V_{1}^{2}-\mu_{V 2} \\
\vdots \\
V_{n}^{2}-\mu_{V 2}
\end{array}\right\}} \\
\hat{V}_{0, i}=\sqrt{\left(x_{i}^{2}-V_{W, X}\right)+\left(y_{i}^{2}-V_{W, Y}\right)}, \quad \hat{V}_{0}=\mu_{V 0}
\end{gathered}
$$

If the system flies in a straight line, the ground track velocity components will be constant and the matrix on the left-handed side of Eq. (23) will contain only measurement noise. The conditioning of the problem is improved by adding measurements from a variety of azimuth angles. Each constant control segment will create a constant turn rate and will contain a continuous string of measurements over a span of heading angles. Insight into the effectiveness of airspeed estimation using the specified technique can be given by assuming that GPS measurements are available at a discrete number of heading angles equally spaced over a span $\Delta \psi$. Equation (23) can be solved in closed form and the propagation of GPS measurement errors can be determined analytically for a given number of measurements. An upper bound on the analytical solutions with as few as three heading measurements can be expressed simply by Eq. (25):

$$
\sigma\left(\hat{V}_{0}\right)<\frac{\sigma\left(V_{\mathrm{GPS}}\right)}{\sin (\delta / 4)^{2}}, \quad \delta=\min (|\Delta \psi|, 2 \pi)
$$

As the heading angle span tends to zero, the airspeed estimate error is unbounded, and as the heading angle span tends to a complete circle, the airspeed estimate error is bounded by the GPS velocity measurement error. If the airspeed error bound obtained from Eq. (25) for a particular flight segment is unacceptably large, the wind estimates from adjacent segments of the same flight can be averaged and used in Eq. (24).

Once the airspeed is determined, steady-state lift, drag and turn rate data can be extracted for each segment of constant control deflection. Based on the vector diagrams in Fig. 4, lift and drag can be calculated from turn rate, airspeed, and descent rate data according to Eqs. (26-28). The steady-state lift and drag data are then converted into coefficient form for matching to simulation:

$$
\begin{gathered}
1 / \tan \gamma=V_{0} / \dot{z}=L^{\prime} / D \\
L^{\prime}=W \cos \gamma \\
L=\sqrt{L^{\prime 2}+\left(m V_{0} \omega\right)^{2}}
\end{gathered}
$$

\section{Matching Simulation Model to Steady-State Data}

The rigging of the parafoil and payload system is fixed, which implies that for a given symmetric brake deflection there exists a single trim angle of attack. This means that the lift and drag vs angleof-attack behavior cannot be estimated from steady-state flight-test data for a typical airdrop system.

Nicolaides [19] conducted extensive wind tunnel tests on airdrop canopies and compiled trends of lift and drag vs angle-of-attack behavior as a function of aspect ratio. The lift and drag vs angle-ofattack coefficients $\left(C_{L A}\right.$ and $\left.C_{D A 2}\right)$ were selected based on these empirical trends. For the canopy element representation, $C_{D A 2}$ is assumed to be constant across the span, while $C_{L A}$ is varied to approximate an elliptical lift distribution while maintaining the lift curve slope of the entire canopy at the desired value. The shape of the lift and drag vs angle-of-attack curves are set according to experimental trends, leaving the level of the lift and drag curves to be set to
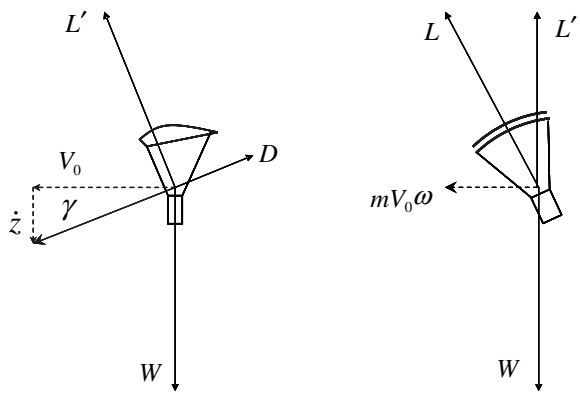

Fig. 4 Relationship of lift and drag to velocity.

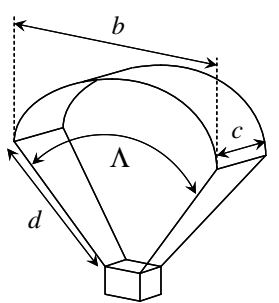

Fig. 5 Canopy geometry parameters. 
match the trim characteristics observed in flight. Three remaining parameters, $C_{L 0}, C_{D 0}$, and the canopy incidence angle are required to determine the trimmed flight condition for the simulation model. For simplicity, $C_{L 0}$ is fixed at zero so that $C_{D 0}$ and the canopy incidence angle can be set to match the trim lift and drag coefficients derived from flight test. The remaining aerodynamic coefficients $\left(C_{L B}, C_{L B 3}\right.$, $C_{D B}$, and $\left.C_{D B 3}\right)$ are associated with steady-state response to brake deflection. These coefficients determine the change in longitudinal trim of the system under symmetric braking as well as the change in lateral "trim" of the system under asymmetric braking. Lateral trim is used here to refer to a flight condition in which the system has reached a steady-state turn rate under an asymmetric brake input. The small fins under the outboard edges of the canopy will also have an effect on the lateral trim condition. The lift and drag characteristics of these fins were fixed, but their area was allowed to vary. The logic behind this choice is that the effective area of the fins can be increased to account for spanwise effects not modeled by the five main panels. This leaves a total of five parameters to be determined to match flighttest data for steady-state lift and drag at different levels of symmetric brake as well as steady-state turn rate at different levels of asymmetric brake.

Longitudinal trim is determined according to Eq. (29), which represents a system of three nonlinear equations (nonlinear due to the relationship between trim velocity, angle of attack, and aerodynamic forces). Lateral trim is determined according to Eq. (30), which represents a system of eight nonlinear equations. Lift coefficient is assumed to vary linearly with angle of attack, so there is only one possible trim condition for a given control input. The quadratic relationship between drag coefficient and angle of attack creates a singularity when angle of attack is zero, so the initial guess for the trim velocity components should result in a positive angle of attack (this implies setting the initial guesses for $u$ and $w$ to positive numbers). In the author's experience, quadratic convergence to the trim condition is achieved from the first iteration if the initial guess for the trim velocity components are set to average values observed in flight test:

$$
\begin{aligned}
& \text { given: }\{\phi, v, p, q, r\}^{T}=0 \\
& \text { find: }\{\theta, u, w\}^{T}, \quad \text { s.t. }\{\dot{\theta}, \dot{u}, \dot{w}\}^{T}=0
\end{aligned}
$$

$$
\text { find: }\{\phi, \theta, u, v, w, p, q, r\}^{T}, \quad \text { s.t. }\{\dot{\phi}, \dot{\theta}, \dot{u}, \dot{v}, \dot{w}, \dot{p}, \dot{q}, \dot{r}\}^{T}=0
$$

A nonlinear set of trim equations can be solved to determine steady-state behavior at a specified control setting for a given set of aerodynamic parameters. The goal of the system identification exercise is to solve a nonlinear regression problem to determine the aerodynamic parameters such that the trim lift, drag, and turn rate vs control input behavior of the simulation model matches flight-test data. Both the nonlinear regression and the nonlinear trim problem are well behaved so the details of the solution procedure are not critical. The nonlinear regression to determine the aerodynamic parameters is performed using the Levenberg-Marquardt method, while the nonlinear trim equations are solved at each iteration of the aerodynamic parameters using the Newton-Raphson method [20].

\section{Experimental Parafoil and Payload System}

Flight tests were conducted with a miniature parafoil and payload system $[21,22]$. The canopy geometry parameters defined in Fig. $\underline{5}$ are listed in Table 1 along with the mass, inertia, and AM properties of the system. The two inputs to the system, left and right brake deflection, modify the aerodynamics of the outer canopy panels. The parameters defining the five main canopy elements and two stabilizing fins are given in Table 2. The payload contains a sensor suite consisting of accelerometers, gyroscopes, a magnetometer, a GPS receiver, and a barometric altimeter.
Table 1 Mass and geometry parameters

\begin{tabular}{ccc}
\hline \hline Parameter & Value & Units \\
\hline$\Lambda$ & 70 & $\mathrm{deg}$ \\
$b$ & 1.77 & $\mathrm{~m}$ \\
$c$ & 0.8 & $\mathrm{~m}$ \\
$d$ & 1.2 & $\mathrm{~m}$ \\
$S_{P}$ & 0.0325 & $\mathrm{~m}{ }^{2}$ \\
Total weight & 2.372 & $\mathrm{~kg}$ \\
$I_{X X}$ & 0.423 & $\mathrm{~kg} \cdot \mathrm{m}^{2}$ \\
$I_{Y Y}$ & 0.401 & $\mathrm{~kg} \cdot \mathrm{m}^{2}$ \\
$I_{Z Z}$ & 0.0529 & $\mathrm{~kg} \cdot \mathrm{m}^{2}$ \\
$I_{X Z}$ & 0.0298 & $\mathrm{~kg} \cdot \mathrm{m}^{2}$ \\
$A$ & 0.02 & $\mathrm{~kg}$ \\
$B$ & 0.13 & $\mathrm{~kg}$ \\
$C$ & 0.64 & $\mathrm{~kg}$ \\
$P$ & 0.011 & $\mathrm{~kg} \cdot \mathrm{m}^{2}$ \\
$Q$ & 0.013 & $\mathrm{~kg} \cdot \mathrm{m}^{2}$ \\
$R$ & 0.006 & $\mathrm{~kg} \cdot \mathrm{m}^{2}$ \\
\hline \hline
\end{tabular}

Table 2 Canopy element definition

\begin{tabular}{ccccccc}
\hline \hline & \multicolumn{3}{c}{ Size } & \multicolumn{2}{c}{ Orientation } & \multicolumn{3}{c}{ Distance from mass center } \\
\cline { 2 - 7 } Element & $S, \mathrm{~m}^{2}$ & $\phi, \mathrm{deg}$ & $\theta, \mathrm{deg}$ & $x, \mathrm{~m}$ & $y, \mathrm{~m}$ & $z, \mathrm{~m}$ \\
\hline 1 & 0.28 & 35 & -18 & 0 & 0.44 & -1.11 \\
2 & 0.28 & 17.5 & -18 & 0 & 0.22 & -1.18 \\
3 & 0.28 & 0 & -18 & 0 & 0 & -1.2 \\
4 & 0.28 & -17.5 & -18 & 0 & -0.22 & -1.18 \\
5 & 0.28 & -35 & -18 & 0 & -0.44 & -1.11 \\
6 & 0.1 & 90 & 0 & -0.5 & 0.8 & -1 \\
7 & 0.1 & -90 & 0 & -0.5 & -0.8 & -1 \\
\hline \hline
\end{tabular}

\section{Results}

The following results were generated by applying the techniques described previously to flight-test data for the microparafoil and payload system. A set of three flights was conducted specifically for system identification purposes and is used for matching the simulation model. A fourth flight conducted on a separate occasion during GNC development is used to validate the identified model.

\section{A. Wind and Airspeed Estimation}

The wind and airspeed estimation technique described previously was applied to GPS data from all of the flights. Figure $\underline{6}$ shows a comparison of the ground track velocity measured during flight 1 with the ground track velocity constructed from the extracted airspeed and wind estimates for each segment of constant control input. The estimates are discontinuous at this stage because the goal is only to extract steady-state data averaged over each segment of constant control input.

Estimated airspeeds for each constant control segment along with the estimated error bound determined by Eq. (25) are listed in Table 3 . Two of the flight segments contained less than a $120 \mathrm{deg}$ variation in heading angle, implying that the airspeed estimation error for these segments may be more than four times the GPS

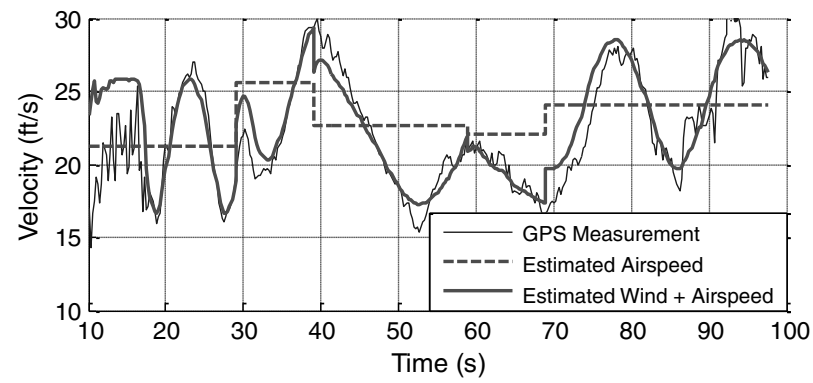

Fig. 6 Example reconstruction of ground track velocity from estimated wind and airspeed. 
Table 3 Airspeed estimate statistics for constant control segments

\begin{tabular}{crcccc}
\hline \hline Flight & \multicolumn{1}{c}{$\delta_{A}$} & $\delta_{B}$ & $\Delta \psi, \operatorname{deg}$ & $\hat{V}_{0}, \mathrm{~m} / \mathrm{s}$ & $\sigma\left(\hat{V}_{0}\right), \mathrm{m} / \mathrm{s}$ \\
\hline 1 & 0.33 & 0.17 & 189 & 7.4 & 0.3 \\
1 & -0.33 & 0.50 & 195 & 7.2 & 0.4 \\
1 & 0.00 & 0.33 & 77 & 6.9 & 0.4 \\
1 & 0.33 & 0.17 & 606 & 7.2 & 0.4 \\
2 & -0.67 & 0.67 & 1010 & 6.7 & 0.3 \\
2 & 0.33 & 0.17 & 141 & 7.3 & 0.7 \\
2 & -0.33 & 0.50 & 202 & 7.2 & 0.5 \\
2 & 0.00 & 0.33 & 112 & 7.1 & 0.5 \\
2 & 0.33 & 0.17 & 371 & 7.3 & 0.5 \\
3 & 0.00 & 1.00 & 360 & 4.0 & 1.2 \\
3 & 1.00 & 0.50 & 208 & 5.8 & 3.4 \\
\hline \hline
\end{tabular}

Table 4 Identified aerodynamic parameters

\begin{tabular}{lccc}
\hline \hline \multicolumn{1}{c}{ Fixed } & & \multicolumn{2}{c}{ Used to match data } \\
\hline Parameter & Value & Parameter & Value \\
\hline$C_{L 0}$ & 0 & Incidence & $-18 \mathrm{deg}$ \\
$C_{L A}$ (overall) & 2 & $C_{D 0}$ & 0.11 \\
$C_{D A 2}$ & 2 & $C_{D B}$ & 0.03 \\
$C_{L B}$ & 0 & $C_{D B 3}$ & 0.1 \\
$C_{L B 3}$ & 0 & $S_{\text {fin }}$ & $0.1 \mathrm{~m}^{2}$ \\
\hline \hline
\end{tabular}

velocity measurement error. For these two flight segments, the airspeed is extracted using an average of the wind estimates from adjacent flight segments. The error estimates for these segments are assumed to be the larger of the error estimates for the adjacent flight segments.

\section{B. Matching Steady-State Lift, Drag, and Turn Rate Data}

The aerodynamic coefficients determined for the simulation model are given in Table 4 . The lift curve slope $\left(C_{L A}\right)$ given in the table is the value for the entire canopy selected to match wind tunnel
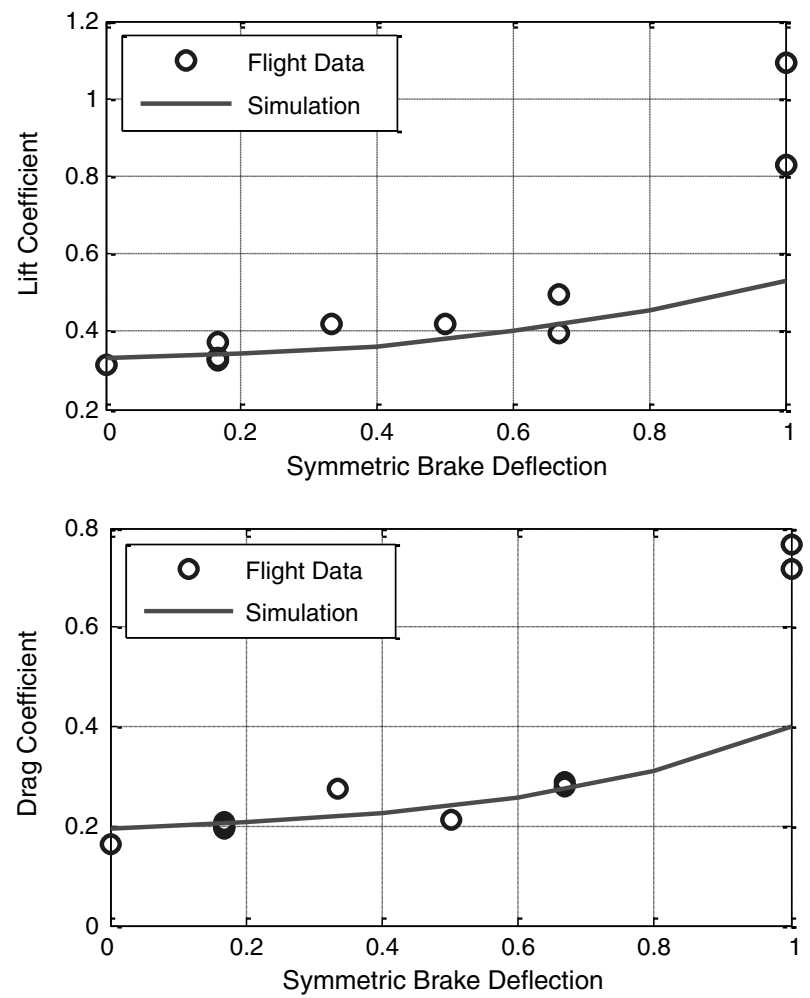

Fig. 7 Matching steady-state lift and drag coefficient vs symmetric brake deflection. data. The lift curve slopes for the five main canopy elements are varied across the span to approximate an elliptical lift distribution $\left(C_{L A, 1-5}=1.4,2.32,2.54,2.32,1.4\right)$. The parameters determining the effect of symmetric braking on the lift coefficient curve $\left(C_{L B}\right.$ and $C_{L B 3}$ ) did not have a significant impact on the matching of the simulation to flight-test data, so they were set to zero. The standard deviation of the matching error between the simulated and observed lift and drag coefficients is on the order of $10 \%$, but the inclusion of these parameters produced only a $0.5 \%$ reduction in the error.

The fin area that produces the best match with the turn rate data is significantly larger than the actual fin area $\left(0.1 \mathrm{vs} 0.04 \mathrm{~m}^{2}\right.$ for the actual fins). The enlarged fin area in the simulation model indicates that there is likely an additional source of directional stability and/or roll and yaw damping that is not modeled by the five main canopy panels.

Figures 7 and 8 compare the lift, drag, and turn rate data extracted from the flight-test data to steady-state values from the identified simulation models. Notice in Fig. 7 that symmetric brake deflection results in an increase in both the steady-state lift and drag coefficients of the system even though the effect of braking is modeled purely as additional drag. This is because the additional drag causes the canopy to trim at a higher angle of attack, producing an overall increase in lift as well as drag. The dramatic increase in lift and drag at high symmetric brake deflection is not matched by the simulation. The problem is that if the brakes are given sufficient control authority to induce such high lift and drag under symmetric braking, such control authority will produce far too much turn rate under asymmetric braking. It seems that the brakes are able to produce a greater effect when deflected together than the sum of their effects when they are deflected separately. Precise modeling of control behavior near stall is not typically a requirement for airdrop simulation, so no attempts were made to match this behavior exactly.

\section{Comparing Time Histories for Validation}

The 6-DOF simulation model using the seven discrete canopy elements is completely specified by matching the steady-state data. IMU data are used purely for validation and was not used during the identification process. All angular rate data displayed in the following charts were passed through a zero-phase, second-order Butterworth filter with a cutoff frequency of $1-5 \mathrm{~Hz}$.

The measurement data contain sensor error, relative motion between the canopy and payload, and excitation from turbulence. These are all sources of noise that will not be matched by a six DOF representation of the system. For this particular system, the transient response of the canopy to a control input takes approximately $1-3 \mathrm{~s}$ to settle; so it is safe to assume that any variation in the measured velocities, orientation angles, and angular rates observed after the first five seconds of a constant control segment are the result of sensor error, relative motion, and turbulence. The level of noise associated with these disturbances can then be estimated simply by computing the standard deviations of the measurements over the portions of each constant control segment after the assumed $5 \mathrm{~s}$ settling period. For comparison, the standard error between the simulated and measured response is also calculated. The estimated noise level $\left(\sigma_{E}\right)$ and

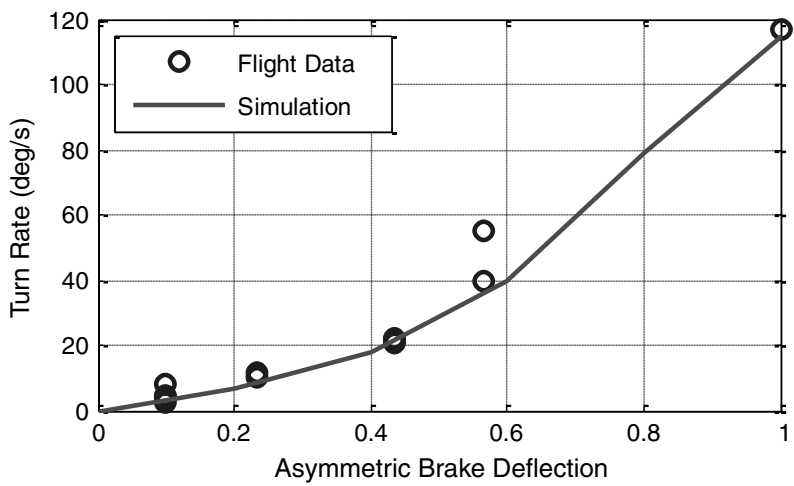

Fig. 8 Matching steady-state turn vs asymmetric brake deflection. 
Table 5 Comparison of matching error to estimated noise levels

\begin{tabular}{lcccccc}
\hline \hline & \multicolumn{3}{c}{ Flight 1 } & \multicolumn{3}{c}{ Flight 6 } \\
\cline { 2 - 7 } State & $\sigma_{E}$ & $\sigma_{M}$ & $\frac{\sigma_{M}}{\sigma_{E}}$ & $\sigma_{E}$ & $\sigma_{M}$ & $\frac{\sigma_{M}}{\sigma_{E}}$ \\
\hline$V_{0}, \mathrm{~m} / \mathrm{s}$ & 0.39 & 0.41 & 1.1 & 2.30 & 2.27 & 1.0 \\
$\dot{z}, \mathrm{~m} / \mathrm{s}$ & 0.20 & 0.54 & 2.7 & 0.53 & 1.40 & 2.6 \\
$\phi, \mathrm{deg}$ & 1.57 & 4.99 & 3.2 & 6.04 & 7.01 & 1.2 \\
$\theta, \mathrm{deg}$ & 1.42 & 2.91 & 2.1 & 5.15 & 6.49 & 1.3 \\
$p, \mathrm{deg} / \mathrm{s}$ & 8.61 & 9.36 & 1.1 & 14.7 & 21.3 & 1.5 \\
$q, \mathrm{deg} / \mathrm{s}$ & 8.59 & 9.75 & 1.1 & 18.0 & 24.5 & 1.4 \\
$r, \mathrm{deg} / \mathrm{s}$ & 11.7 & 12.6 & 1.1 & 26.7 & 28.6 & 1.1 \\
\hline \hline
\end{tabular}

standard matching error $\left(\sigma_{M}\right)$ are obtained for every response for each constant control segment according to Eq. (31):

$$
\begin{aligned}
\sigma_{E} & =1 / n \sqrt{\sum_{i=1, n}\left(y_{i, \mathrm{MEAS}}-\bar{y}_{\mathrm{MEAS}}\right)^{2}} \\
\sigma_{M} & =1 / n \sqrt{\sum_{i=1, n}\left(y_{i, \mathrm{SIM}}-y_{i, \mathrm{MEAS}}\right)^{2}}
\end{aligned}
$$

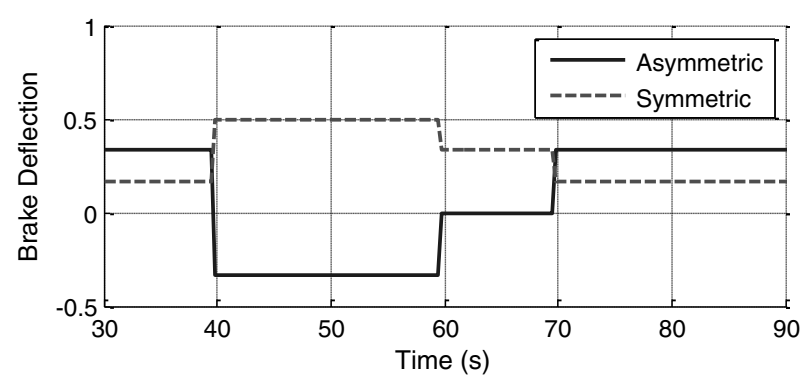

Fig. 9 Control inputs for flight 1.
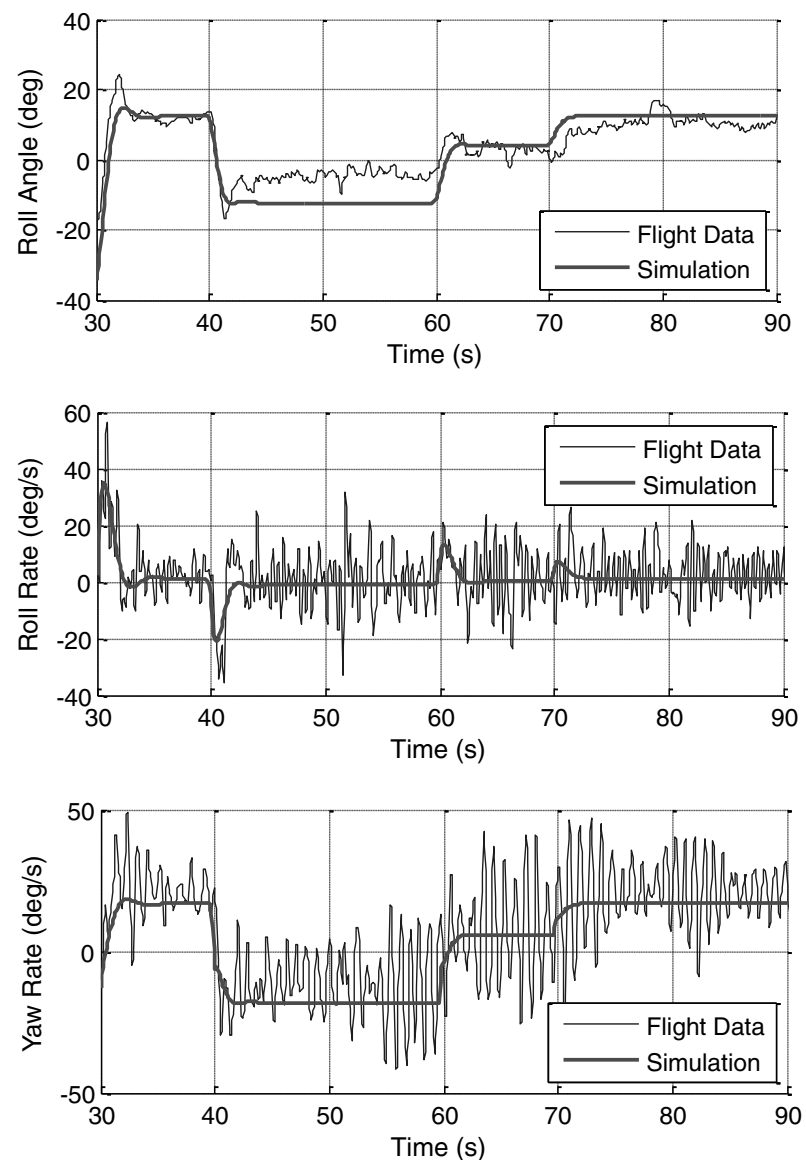

Fig. 10 Simulation comparison to IMU data for flight 1.
The ratio of the matching error to the estimated noise provides a convenient way of estimating the quality of the simulation model. A ratio of one means that the error between the simulation model and the measurements is of the same magnitude as unmodeled disturbances, implying that the simulation model provides an excellent reproduction of the system dynamics. The matching error, estimated noise levels, and the ratio between the two are reported for each flight 1 and flight 3 in Table 5. These values are averages for each flight of the estimates compute from the individual flight segments. Flight 1 contains only moderate variations in symmetric brake deflection and is appropriate for evaluating the lateral dynamics of the simulation model. Flight 3 contains a large symmetric brake input for evaluating the longitudinal dynamics and also a spiral turn for evaluating the ability of the simulation model to capture highly nonlinear behavior.

Referring to Table 5, the ratio of matching error to measurement noise for forward velocity, descent rate, and yaw rate should all be close to one because the simulation model was indirectly matched to the steady-state values of these measurements (indirect in the sense that forward airspeed and descent rate were converted to lift and drag, and yaw rate of the payload is only approximately equivalent to turn rate when the Euler angles of the payload are small). This is the case for the forward airspeed and yaw rate, but the matching error for descent rate is quite large. This is because the descent rate behavior was not repeatable from flight to flight, most likely due to significant variations in the vertical wind component caused by thermal activity. By comparing repeated applications of the same control input during different flights, the standard deviation of the variation in descent rate from flight to flight was estimated to be $1.02 \mathrm{~m} / \mathrm{s}$, which fits nicely with the observed matching error. The ratios of matching error to measurement noise for the other states are all close to 1 as well, with the exception of the roll and pitch angles for the first flight. However,

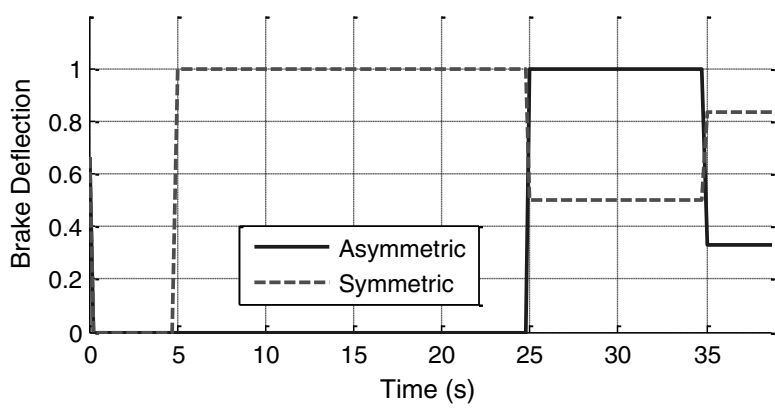

Fig. 11 Control inputs for flight 3.
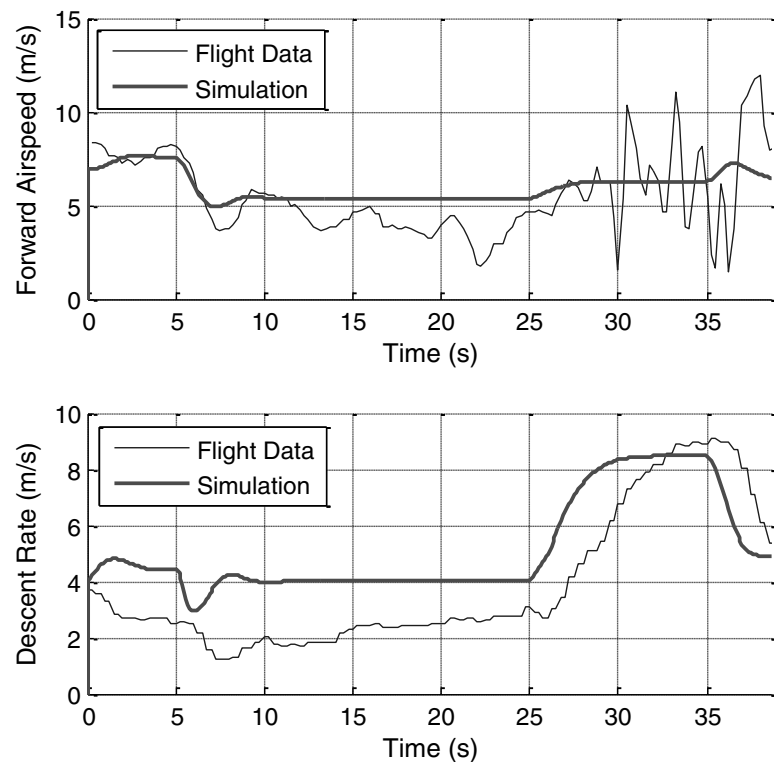

Fig. 12 Velocity comparisons for flight 3. 

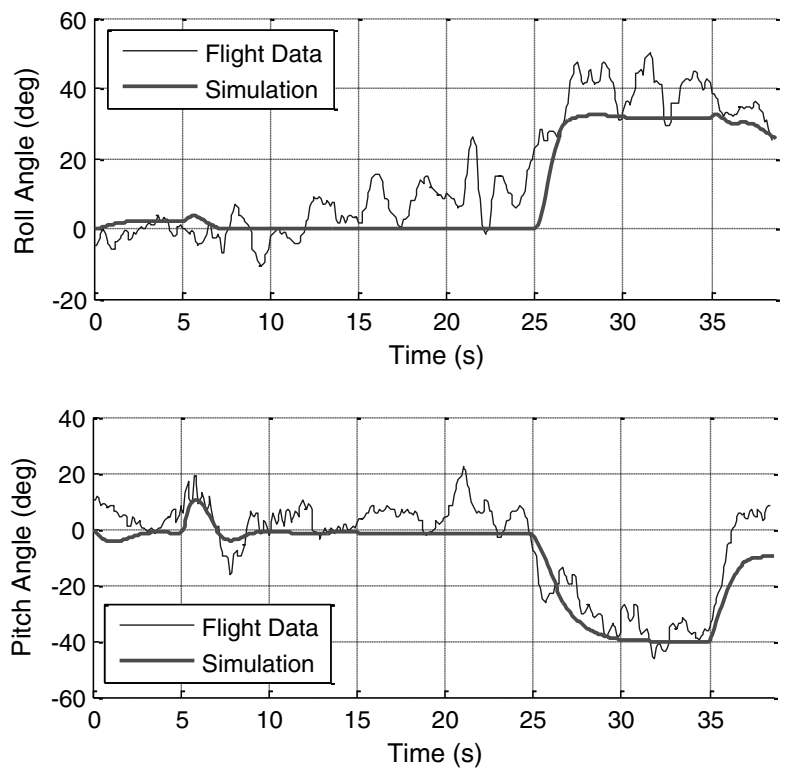

Fig. 13 Euler angle comparisons for flight 3.

the actual values of the matching error are on the order of only $5 \mathrm{deg}$. This implies that the simulation model based only on steady-state GPS data is able to reproduce the dynamic pitch and roll response of the actual system to an accuracy on the order of $5 \mathrm{deg}$.

\section{Comparison of Time Histories for Flight 1}

Figure 9 shows the control input sequence for flight 1 and Fig. 10 compares the lateral dynamic response from the simulation model with the measured data. The yaw-roll coupling seems to be very well captured given that the simulation model was created based only on steady-state data derived from GPS measurements. This indicates that the simple canopy aerodynamic model based on seven elements producing only lift and drag is able to reproduce the relevant features of the parafoil dynamics.

\section{Comparison of Time Histories for Flight 3}

The control input sequence for flight 3 is shown in Fig. 11. The application of full brakes at $5 \mathrm{~s}$ represents a flare and is purely a longitudinal maneuver. At $25 \mathrm{~s}$, the controls transition to full right brake, initiating a tight, spiral turn. Figure $\underline{12}$ compares airspeed and
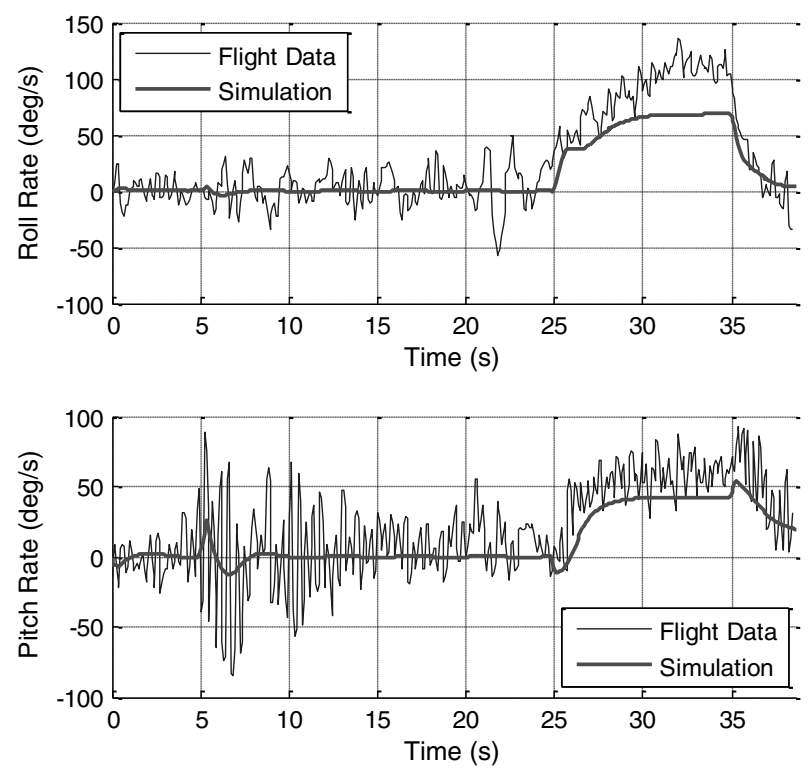

Fig. 14 Angular rate comparisons for flight 3.

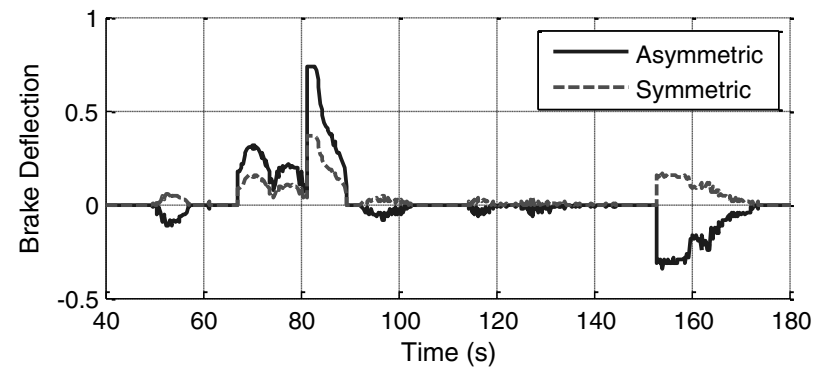

Fig. 15 Control inputs (compensated for bias) for validation flight.

descent rate, Fig. 13 compares roll and pitch angle, and Fig. 14 compares the roll and pitch rate response of the simulation model to the flight-test data. The amplitudes and frequency responses of the simulation are all well within an order of magnitude of the observations for both the flare and the spiral turn maneuvers. The descent rate comparison suffers from large disturbances to descent rate from thermal activity mentioned previously as well as the inability to match the dramatic change in lift and drag coefficient at high brake deflection mentioned in the discussion of the steady-state matching. The flare at $5 \mathrm{~s}$ induces a pitch oscillation. The dynamic response of the simulation model in velocity and pitch is similar in both amplitude and frequency to the observed oscillation.

The simulation is also able to adequately capture the coupling between the lateral and longitudinal dynamics during the spiral turn. It is important to note that the spiral turn is an extreme maneuver in a highly nonlinear dynamic region that the system would be unlikely to enter during normal flight operations. The fact that the simulation model shows qualitative agreement to the flight-test data during this maneuver is an indication that the simple canopy aerodynamic model is able to reproduce complex, nonlinear features of the parafoil dynamics.
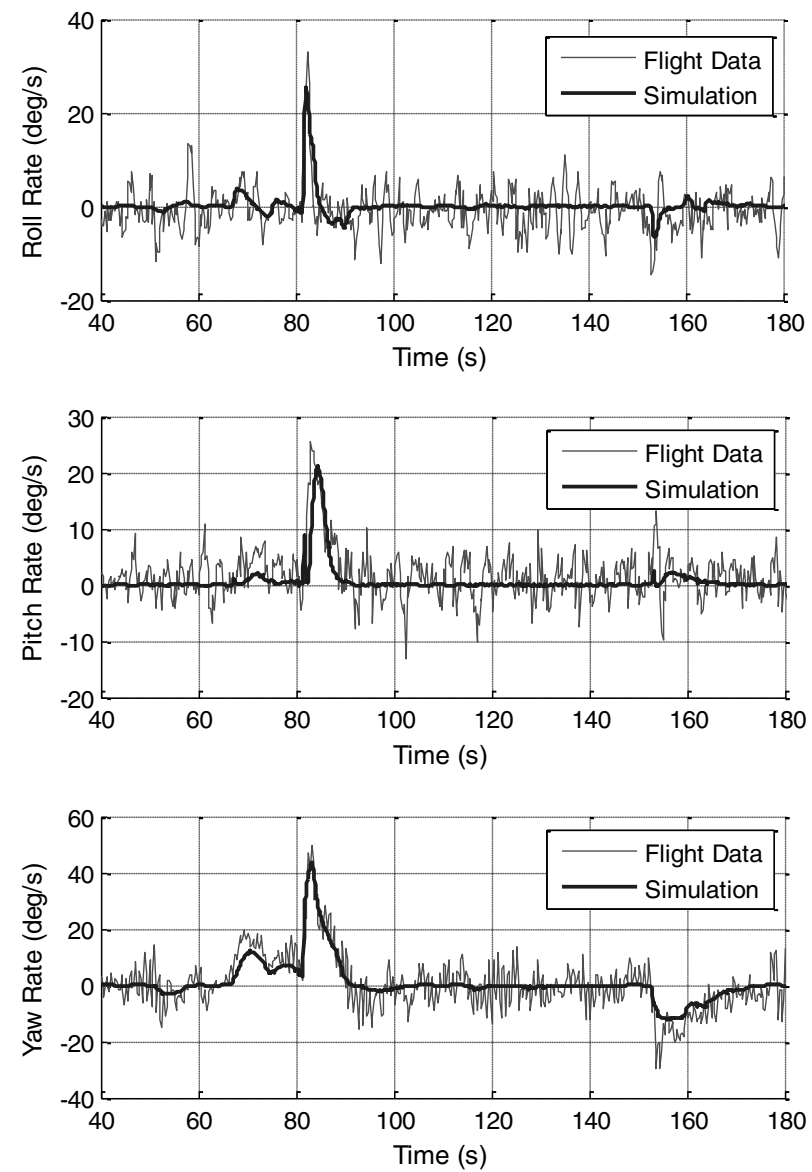

Fig. 16 Angular rate comparisons for validation flight. 


\section{Model Validation}

To validate the identified model, simulation results are compared to data from a flight test that was not used during the system identification process. The actual canopy and payload were different for this flight, though they were both made to the same specifications. The recorded control inputs for this canopy were adjusted to account for a control bias (36\% left control required to fly straight). The control inputs for this flight with the control bias removed are shown in Fig. 15. Comparisons between the flight data and simulation data using the identified aerodynamic model are shown in Fig. 16. The identified model is in good agreement with this separate set of flight data, indicating that the system identification procedure was able to produce a valid representation of this parafoil and payload system.

\section{Conclusions}

A specialized system identification method for parafoil and payload air vehicles using only GPS data is reported here that has been found to be very robust and reliable in practice. The proposed method uses a two-phase approach beginning with a wind estimation procedure to extract steady-state flight characteristics followed by an aerodynamic parameter identification to match these steady-state characteristics rather than the flight-test data directly. These steadystate control characteristics are averaged over an extended number of measurements, reducing the impact of sensor noise and turbulence so that high-quality estimates can be obtained. Using only GPS data from the flight test, a full 6-DOF dynamic simulation model is generated. A simplified aerodynamic representation of the canopy that requires identification of only steady-state vehicle response to control inputs enables complete definition of the dynamic model. Since the system identification method only needs steady-state response of the aircraft due to a set of constant control inputs, the flight-test procedure is straightforward and can be performed in a manual mode or autonomously with an autopilot. While the simulation model is created entirely from steady-state data, the simple, physics-based representation of the canopy allows even highly nonlinear dynamic behavior to be reproduced accurately in simulation.

\section{Acknowledgments}

The authors would like to thank Stacey Keith and the people from Skydive Alabama as well as Harwood Drake of Cullman Airport for their assistance in conducting the flight tests.

\section{References}

[1] Etkin, B., Dynamics of Atmospheric Flight, Wiley, New York, 1972.

[2] Klein, V., and Morelli, E., Aircraft System Identification: Theory and Practice, AIAA Education Series, AIAA, Reston, VA, 2006.

[3] Jategaonkar, R. V., Flight Vehicle System Identification: A Time Domain Approach, AIAA Education Series, AIAA, Reston, VA, 2006.

[4] Jann, T., Doherr, K., and Gockel, W., "Parafoil Test Vehicle ALEX: Further Development and Flight Test Results," 15th CEAS/AIAA Aerodynamic Decelerator Systems Technology Conference, AIAA Paper 1999-1751, Toulouse, France, 8-11 June 1999.

[5] Jann, T., "Aerodynamic Model Identification and GNC Design for the Parafoil-Load System ALEX," 16th AIAA Aerodynamic Decelerator Systems Technology Conference and Seminar, AIAA Paper 20012015, Boston, MA, 21-24 May 2001.

[6] Jann, T., "Aerodynamic Coefficients for a Parafoil Wing with Arc Anhedral: Theoretical and Experimental Results," 17th AIAA Aerodynamic Decelerator Systems Technology Conference and
Seminar, AIAA Paper 2003-2106, Monterey, CA, 19-22 May 2003.

[7] Jann, T., and Strickert, G., "System Identification of a Parafoil-Load Vehicle: Lessons Learned," 18th AIAA Aerodynamic Decelerator Systems Technology Conference and Seminar, AIAA Paper 20051663, Munich, 23-26 May 2005.

[8] Hur, G., and Valasek, J., "System Identification of Powered ParafoilVehicle from Flight Test Data," Proceedings of the AIAA Atmospheric Flight Mechanics Conference, AIAA Paper 2003-5539, Austin, TX, 11-14 Aug. 2003.

[9] Valasek, J., and Chen, W., "Observer/Kalman Filter Identification for Online System Identification of Aircraft," Journal of Guidance, Control, and Dynamics, Vol. 26, No. 2, March-April 2003, pp. 347 353.

doi: $10.2514 / 2.5052$

[10] Kothandaraman, G., and Rotea, M., "Simultaneous-PerturbationStochastic-Approximation Algorithm for Parachute Parameter Estimation," Journal of Aircraft, Vol. 42, No. 5, Sept.-Oct. 2005, pp. 1229-1235.

[11] Yakimenko, O., and Statnikov, R., "Multicriteria Parametrical Identification of the Parafoil-Load Delivery System," 18th AIAA Aerodynamic Decelerator Systems Technology Conference and Seminar, AIAA Paper 2005-1664, Munich, 23-26 May 2005.

[12] Carter, D., George, S., Hattis, P., Singh, L., and Tavan, S., "Autonomous Guidance, Navigation, and Control of Large Parafoils," 18th AIAA Aerodynamic Decelerator Systems Technology Conference and Seminar, AIAA Paper 2005-1643, Munich, 23-26 May 2005.

[13] Carter, D., George, S., Hattis, P., McConley, M., Rasmussen, S., Singh, L., and Tavan, S., "Autonomous Large Parafoil Guidance, Navigation, and Control System Design Status," 19th AIAA Aerodynamic Decelerator Systems Technology Conference and Seminar, AIAA Paper 2007-2514, Williamsburg, VA, 21-24 May 2007.

[14] Carter, D., Singh, L., Wholey, L., Rasmussen, S., Barrows, T., George, S., McConley, M., Gibson, C., Tavan, S., and Bagdonovich, B., "BandLimited Guidance and Control of Large Parafoils," 20th AIAA Aerodynamic Decelerator Systems Technology Conference and Seminar, AIAA Paper 2009-2981, Seattle, WA, 4-7 May 2009.

[15] Majji, M., Juang, J., and Lunkins, J., "Time-Varying Eigensystem Realization Algorithm," Journal of Guidance, Control, and Dynamics, Vol. 33, No. 1, Jan.-Feb. 2010, pp. 13-28. doi: $10.2514 / 1.45722$

[16] Majji, M., Juang, J., and Lunkins, J., "Observer/Kalman-Filter TimeVarying System Identification," Journal of Guidance, Control, and Dynamics, Vol. 33, No. 3, May-June 2010, pp. 887-900. doi: $10.2514 / 1.45768$

[17] Slegers, N., and Costello, M., "Aspects of Control for Parafoil and Payload System," Journal of Guidance, Control, and Dynamics, Vol. 26, No. 6, Nov.-Dec. 2003, pp. 898-905. doi: $10.2514 / 2.6933$

[18] Lissaman, P., and Brown, G., "Apparent Mass Effects on Parafoil Dynamics," 12th AIAA Aerodynamic Decelerator Systems Technology Conference and Seminar, AIAA Paper 1993-1236, London, 1013 May 1993.

[19] Nicolaides, J., "Parafoil Wind Tunnel Tests," U.S. Air Force Flight Dynamics Lab., AFFDL-TR-70-146, June 1971, Wright-Patterson $\mathrm{AFB}, \mathrm{OH}$.

[20] Press, W., Teukolsky, S., Vetterling, W., and Flannery, B., Numerical Recipes in Fortran 77, 2nd ed., Cambridge Univ. Press, New York, 1992, pp. 15-5, Chap. 9.6.

[21] Yakimenko, O., Slegers, N., and Tiaden, R., "Development and Testing of the Miniature Aerial Delivery System Snowflake," 20th AIAA Aerodynamic Decelerator Systems Technology Conference and Seminar, AIAA Paper 2009-2980, Seattle, WA, 4-7 May 2009.

[22] Slegers, N., and Yakimenko, "Terminal Guidance of Autonomous Parafoils in High Wind to Airspeed Ratios," Proceedings of the Institution of Mechanical Engineers. Part G, Journal of Aerospace Engineering, Vol. 225, No. 3, March 2011, pp. 336-346. doi:10.1243/09544100JAERO749 